\title{
Transferrin-functionalized nanographene oxide for delivery of platinum complexes to enhance cancer- cell selectivity and apoptosis-inducing efficacy
}

This article was published in the following Dove Press journal:

International Journal of Nanomedicine

13 July 2017

Number of times this article has been viewed

Hai Zhu'

Binwei Zhou ${ }^{2}$

Leung Chan²

Yanxin Du'

Tianfeng Chen'

'Department of Internal Medicine and Orthopedics, Guangdong Provincial Hospital of Traditional Chinese Medicine, ${ }^{2}$ Department of Chemistry, Jinan University, Guangzhou, China

Correspondence: Tianfeng Chen;

Yanxin Du

Department of Internal Medicine and Orthopedics, Guangdong Provincial

Hospital of Traditional Chinese Medicine,

I I I Dade Road, Guangzhou, Guangdong

5I0I20, China

Email tchentf@jnu.edu.cn;

I3828456388@I26.com
Abstract: Rational design and construction of delivery nanosystems for anticancer metal complexes is a crucial strategy to improve solubility under physiological conditions and permeability and retention behavior in tumor cells. Therefore, in this study, we designed and synthesize a transferrin (Tf)-conjugated nanographene oxide (NGO) nanosystem as a cancer-targeted nanocarrier of Pt complexes (Tf-NGO@Pt). This nanodelivery system exhibited good solubility under physiological conditions. Moreover, Tf-NGO@Pt showed higher anticancer efficacy against MCF human breast cancer cells than the free Pt complex, and effectively inhibited cancer-cell migration and invasion, with involvement of reactive oxygen species overproduction. In addition, nanolization also enhanced the penetration ability and inhibitory effect of the Pt complex toward MCF7 breast cancer-cell tumor spheroids. The enhancement of anticancer efficacy was positively correlated with increased cellular uptake and cellular drug retention. This study provides a new strategy to facilitate the future application of metal complexes in cancer therapy. Keywords: cancer targeting, nanographene oxide, nanomedicine, transferrin, platinum complex, cell apoptosis

\section{Introduction}

Cancer has become the leading threat to human health, and there are currently no effective treatments for most kinds of cancer. ${ }^{1}$ Chemotherapy is a common treatment for cancer, and Pt complexes have shown potential utility for chemotherapy and photodynamic therapy. Pt-based antineoplastic agents, such as cisplatin, carboplatin, and oxaliplatin, are now in routine clinical practice. These heavy-metal agents possess bioactivity for treatment of various tumors; however, they have limitations, including gastrointestinal symptoms, renal tubular injury, neuromuscular complications, and ototoxicity. ${ }^{2}$ Cisplatin is a commonly used clinical drug for cancer treatment, but acquired resistance limits it clinical application. As an anticancer agent, platinum has equal or greater antitumor activity and lower toxicity than other metallic cytotoxic compounds to extend the spectrum of activity of metal-based drugs.

In the past 30 years, many Pt complexes have been synthesized and tested for potential antitumor activity. Cisplatin, a typical clinical cancer chemotherapeutic, has been widely prescribed for the treatment of multifarious tumors. Till now, thousands of $\mathrm{Pt}$ analogs have been designed and synthesized in order to treat cancer, with higher activity and more compliance. Besides Pt complexes, Ru is also another transition metal of the Pt group that generally has lower toxicity compared to cisplatin, because of specific targeting and accumulation of drugs in cancer tissues. However, clinical application 
of Pt complexes has been hindered by poor solubility under physiological conditions and low permeability retention in tumor cells. Graphene nanoparticles with oxidized form (nanographene oxides [NGOs]) have higher hydrophilicity than NGO, and are sufficiently beneficial to become a drug-delivery system in vivo. ${ }^{3-7} \mathrm{NGO}$ has been reported as a metal-complex nanoplatform. ${ }^{8-11}$ Conjugation of cancertargeted ligands on the surface of a nanosystem is an effective strategy to enhance tumor selectivity by active targeting. ${ }^{1-13}$ This contributes significantly to achieving maximum anticancer effect and significantly reducing side effects. ${ }^{14-16}$

This study reports on the design of a transferrin (Tf)conjugated NGO nanosystem, which is a specific targeting molecule for oncotherapy. ${ }^{17-19}$ Tf-NGO@Pt nanoparticles exhibit good water solubility through polyethylenimine (PEI) decoration. ${ }^{20}$ Therefore, they offer a solution to the poor solubility of Pt complexes. Based on this design, we found that Tf-NGO@Pt shows higher anticancer efficacy against MCF human breast cancer cells than the free-Pt complex, and can effectively inhibit cancer-cell migration and invasion by induction of cell apoptosis through upregulation of reactive oxygen species (ROS), important biochemical signaling mediators. ${ }^{21-24}$ In addition, nanolization also enhances the penetration ability and inhibitory effect of the Pt complex toward MCF7 breast cancer-tumor spheroids. The enhancement of anticancer efficacy is positively correlated with the increased amount of cellular uptake and cellular drug retention. This study provides a new strategy to facilitate the future application of metal complexes in cancer therapy.

\section{Materials and methods Synthesis of Pt complex and preparation of NGO nanosystem}

The Pt complex (Pt [10-methyldipyridophenazine] $\mathrm{Cl}_{2}$ ) was synthesized according to our previous method. ${ }^{30}$ NGOs were prepared according to a slightly modified Hummer protocol with modification. ${ }^{25-28}$ For synthesis of the NGO nanosystem, the Pt-complex solution $(1 \mathrm{mg} / \mathrm{mL})$ was dropped in $10 \mathrm{~mL} \mathrm{NGO}$ solution $(1 \mathrm{mg} / \mathrm{mL})$ and ultrasound treated for 15 minutes. The product was allowed to react for 12 hours and filtered with water to remove superfluous Pt complex. PEI solution $(2.5 \mathrm{mg} / \mathrm{mL})$ was mixed with the as-prepared solution (NGO-Pt) for 12 hours, and the mixture was dialyzed to remove redundant PEI.

\section{Tf conjugation and characterization}

For Tf conjugation, 0.2 M 1-ethyl-3-(3-dimethylaminopropyl) carbodiimide hydrochloride (EDC) solution was carefully stirred with $\operatorname{Tf}(5 \mathrm{mg} / \mathrm{mL})$ and activated the $\mathrm{Tf}$ amine group for 2 hours. An added NGO-complex PEI solution then stood at $4^{\circ} \mathrm{C}$ overnight. Finally, reagents were removed by dialysis for 8 hours. Tf-NGO@Pt was analyzed with atomic-force microscopy (Bioscope Catalyst NanoScope V), transmission electron microscopy (Hitachi H-7650) with 80 $\mathrm{kV}$ accelerating voltage, and scanning electron microscopy (EX-250; Horiba). A Zetasizer Nano ZS particle analyzer (Malvern Instruments, Malvern, UK) was used to measured size distribution and $\zeta$-potential of nanoparticles. Chemical construction of the nanosystem was characterized by ultraviolet (UV)-visible spectroscopy (Carry 5000) and Fouriertransform infrared (FTIR) spectroscopy (Equinox 55). A bicinchoninic acid kit (Pierce) was used to measure the concentration of Tf conjugate in the nanosystem. Pt content was determined as previously described by inductively coupled plasma atomic emission spectroscopy (ICP-AES). ${ }^{12,29,30}$

\section{Cell lines and cell culture}

Various human cancer-cell lines and normal human cell lines were utilized in this study, including HeLa, CaSki, SiHa, MCF7, Chem5, HepG2 hepatocellular carcinoma, human osteosarcoma MG63, L02 human liver, SV-HUVC1, and A375 cells, obtained from the American Type Culture Collection (Manassas, VA, US). The DMEM media was blended with 50 units/mL of streptomycin (Sigma-Aldrich; St. Louis, MO, USA), 100 units/mL of penicillin (Sigma-Aldrich), and $10 \%$ fetal bovine serum. All cell lines were cultured in $5 \% \mathrm{CO}_{2}$ in a fully humidified atmosphere at $37^{\circ} \mathrm{C} .{ }^{31}$

\section{Tf-NGO@Pt cellular uptake}

The concentration of intracellular complexes was determined by ICP-AES. MCF7 and SV-HUVC1 cells were seeded at $3 \times 10^{6}$ cells per well in six-well plates for 24 hours. The cells were added to Pt complexes and Tf-NGO@Pt (20 $\mu \mathrm{M})$ for different times. The cells were detached with trypsin EDTA solution $\left(5\right.$ minutes, $37^{\circ} \mathrm{C}$ ) and centrifuged to collect the cells. Complex concentration was measured using ICP-AES. The collections were added to mixed acid (concentrated nitric acid:perchloric acid, 3:1) and digested with an infrared (IR) rapid-digestion system (Gerhardt, Königswinter, Germany) at $180^{\circ} \mathrm{C}$ for 2 hours.

\section{Cellular retention assay}

Cellular retention of the Tf-NGO@Pt was quantified using a fluorescence microplate reader. ${ }^{32} \mathrm{MCF} 7$ and SV-HUVC1 cells were seeded at $2 \times 10^{6}$ cells per $\mathrm{mL}(2 \mathrm{~mL})$ and cultured in sixwell plates. Then, the medium was replaced by Pt complexes and Tf-NGO@Pt $(20 \mu \mathrm{M})$ for 8 hours. The medium was then 
removed and replaced with medium $(2 \mathrm{~mL})$ without phenol. After different periods, medium samples $(100 \mu \mathrm{L})$ were removed to measure the fluorescence intensity of cell lysis.

\section{Transferrin competing assay}

Excess Tf receptors (TfRs) were used to bind the TfRs of CMF7 cells to contend with Tf-NGO@Pt. MCF7 cells $\left(100 \mu \mathrm{L}, 7 \times 10^{4}\right.$ cells $\left./ \mathrm{mL}\right)$ were incubated in 96-well plates. After 24 hours, the cells were bound with excess $\mathrm{Tf}$ of $0-5 \mathrm{mg} / \mathrm{mL}$ and incubated for 1 hour at $37^{\circ} \mathrm{C}$, then $20 \mu \mathrm{M}$ of coumarin-6-loaded Tf-NGO@Pt was added. After 2-hour further incubation, cells were rinsed three times with warm PBS and the fluorescence intensity of cell lysis detected using a fluorescence microplate reader.

\section{Tf-NGO@Pt intracellular trafficking}

Intracellular localization of Tf-NGO@Pt was characterized by fluorescence microscopy (IX51; Olympus). ${ }^{33}$ We incubated MG-63 cells (80 nM) of LysoTracker DND-99 (SigmaAldrich) in $2 \mathrm{~cm}$ incubator dishes for 2 hours until $70 \%$ staining confluence had been reached. Then, $1 \mathrm{mg} / \mathrm{mL}$ of DAPI H33258 (Sigma-Aldrich) was added for 30 minutes, cells rinsed with PBS three times and incubated with $20 \mu \mathrm{M}$ coumarin-6-loaded Tf-NGO@Pt for different periods, and observed by fluorescence microscopy.

\section{Cellular uptake mechanisms}

We incubated 7,000 MCF7 cells per well in 96-well plates. ${ }^{34}$ After 24 hours, the cells were incubated with endocytosis inhibitors for 1 hour, while nystatin (Sigma-Aldrich) was cultured for 30 minutes. Subsequently, the cells were cultured with $20 \mu \mathrm{M}$ of coumarin-6-loaded Tf-NGO@Pt for 3 hours. Specific endocytosis-inhibitor concentrations included nystatin $10 \mathrm{mg} / \mathrm{mL}, 80 \mathrm{mM}$ dynasore (Sigma-Aldrich), $0.45 \mathrm{M}$ sucrose, $50 \mathrm{mM}$ 2-deoxy-D-glucose (Sigma-Aldrich), and $10 \mathrm{mM}$ sodium azide $\left(\mathrm{NaN}_{3}\right){ }^{35}$ The cells were cultivated with fresh medium at $4^{\circ} \mathrm{C}$ and treated with coumarin-6loaded Tf-NGO@Pt for a further 3 hours.

\section{Tf-NGO@Pt drug release}

To examine the drug release ofTf-NGO@Pt,10mg Tf-NGO@ Pt was suspended in $10 \mathrm{~mL}$ PBS solution to imitate the different conditions of microenvironment, blood ( $\mathrm{pH} 7.4)$, tumor microenvironment ( $\mathrm{pH}$ 6.8), lysosomes ( $\mathrm{pH} 5.3, \mathrm{pH} 5.3$ with lysozyme), and cellular environment (cell-lysis solution). Samples were constantly waggled in tubes at $37^{\circ} \mathrm{C}$ without illumination. At specific times, known buffer volumes were withdrawn from the tubes and replenished with fresh buffer.
The collections were separated by centrifugation and the supernatants measured by fluorescence microplate reader.

\section{MCF7 tumor spheroids}

MCF7 spheroids were cultured in vitro with a liquid-overlay system. Agarose PBS solution (2\% w:v) was prepared at $80^{\circ} \mathrm{C}$ for 30 minutes, then sterilized using autoclave. The agarose solution $(50 \mu \mathrm{L})$ was covered in the 96-cell plates, then seeded with MCF7 cells (4,000 cells per well). On the first day, the plates were then gently agitated at set intervals and grown for 5 days.

\section{Tf-NGO@Pt penetration ability into MCF7 spheroids and inhibitory effects}

To compare the penetration ability of complexes $(20 \mu \mathrm{M})$ and Tf-NGO@Pt (20 $\mu \mathrm{M})$, the coumarin-6-labeled nanoparticles were added to the MCF7 spheroids and incubated for 12 hours. The tumor spheroids were subsequently scanned at different layers from the spheroid to the middle using confocal laser-scanning fluorescent microscopy (Zeiss LSM700).

Tumor-spheroid size was used to evaluate the cytotoxicity of complexes $(20 \mu \mathrm{M})$ and Tf-NGO@Pt $(20 \mu \mathrm{M})$ using an inverted microscope over 5 days. The major $\left(\mathrm{d}_{\max }\right)$ and minor $\left(\mathrm{d}_{\min }\right)$ diameters of each spheroid were determined, and spheroid volume was calculated as $\mathrm{V}=\left(\pi \times \mathrm{d}_{\max } \times \mathrm{d}_{\text {min }}\right) / 6$. The volume-change ratio for MCF7 spheroids was calculated as $\mathrm{R}=\left(V_{\text {dayl }} / V_{\text {day } 0}\right) \times 100 \%$. Each assay was repeated three times, with sextuplicate determinations for each dose level.

\section{Cell-migration assay}

MCF7 cells $\left(30 \times 10^{4}\right.$ cells $\left./ \mathrm{mL}\right)$ were seeded into six-well plates. Then, the medium was removed and cultured with $3 \%$ fetal bovine-serum medium for a further 6 hours. Subsequently, the cells were wounded by pipette tips, and drugs were added to the plate. Then, 4',6-diamidino-2-phenylindole (DAPI) was added to label the cells and fluorescent microscopy was used to photograph the migrated cells.

\section{Cell-invasion assay}

MCF7 cells $\left(6 \times 10^{4}\right)$ containing the drugs were seeded on the upper side of Matrigel-coated culture insert ( $8 \mu \mathrm{M}$; Corning, NY, USA). After 24-hour cultivation, cotton wipes were used to remove the unmigrated cells on the upper side of the transwell membrane, and methanol was used to fix invaded cells for 10 minutes. After cells had been stained with Giemsa solution, they were observed with the Olympus inverted microscope. As ratio to control samples (\%), quantitative data were measured by manual counting and recorded. 


\section{MTT assay}

As discussed elsewhere, ${ }^{36}$ cell viability is an important factor to evaluate the activity of a nanosystem by detecting the ability of cells to transform MTT to a purple formazan dye..$^{37,38}$ Different cells $\left(2 \times 10^{4}\right.$ cells $\left./ \mathrm{mL}\right)$ were incubated in 96-well plates and cultivated, then different concentrations of Tf-NGO@Pt were added at different times. Subsequently, the cells was incubated with $20 \mathrm{~mL}$ PBS solution with MTT for 5 hours. Finally, the medium was replaced by $200 \mu \mathrm{L}$ dimethyl sulfoxide to dissolve purple formazan and UV absorption at $570 \mathrm{~nm}$ measured to assess cell viability.

\section{Flow cytometry}

Changes in cell-cycle distribution were measured by flow cytometry. ${ }^{39-41}$ Cells were collected and washed three times, then held in precooled $70 \%$ ethanol at $-20^{\circ} \mathrm{C}$ overnight. Fixed cells were incubated with propidium iodide. After being dyed for 1 hour, the cell-cycle distribution for treated cells was measured by flow cytometry (Epics XL; Beckman Coulter, Brea, CA, USA) using MultiCycle software to analyze the data.

\section{TUNEL-DAPI costaining assay}

DNA fragmentation in MCF7 and SV-HUVC1 cells caused by Tf-NGO@Pt was confirmed by terminal deoxynucleotidyl transferase dUTP nick end labeling (TUNEL) assay. Tf-NGO@Pt was added to MCF7 and SV-HUVC1 cells and incubated for 24 hours. Cells were blended with formaldehyde (3.7\%) for 10 minutes and incubated with Triton X-100 $(0.1 \%)$. Cells were then incubated with TUNEL reaction mixture for 1 hour, then $1 \mathrm{mg} / \mathrm{mL}$ DAPI was added at $37^{\circ} \mathrm{C}$. After 15 minutes, cells were rinsed with PBS and measured by fluorescence microscopy (Nikon Eclipse 80i). ${ }^{42}$

\section{Intracellular reactive oxygen species generation}

Production of intracellular ROS in cells induced by TfNGO@Pt was measured by fluorescence intensity in cells, which were stained with dihydroethidium (DHE; Beyotime, Jiangsu, People's Republic of China). ${ }^{42}$ The collected cells were rinsed three times and resuspended in PBS, and next the cells were incubated with DHE $(10 \mu \mathrm{M})$ at $37^{\circ} \mathrm{C}$. After 30 minutes, the various concentrations of Tf-NGO@Pt were added to cells for different periods. Finally, the level of intracellular ROS production was monitored with a microplate reader at 300 and $610 \mathrm{~nm}$.

\section{Statistical analysis}

All experiments were carried out at least in triplicate, and results are expressed as means \pm SD. SPSS was used for statistical analysis. Statistical significance between two groups was analyzed using $P<0.05$ or $P<0.01$. Bars with different characters show statistical significance at $P<0.05$.

\section{Results and discussion Tf-NGO@Pt design, preparation, and characterization}

Figure 1A shows the design chart of the smart nanosystem Tf-NGO@Pt. The preparation of carboxylated NGO was modified from Hummer's method. As shown in Figure 1B, the width of Tf-NGO@Pt was approximately $40 \mathrm{~nm}$. On the other hand, the morphology of Tf-NGO@Pt remained planar. Nanoparticle $\zeta$-potential changes were recorded at different stages. Figure $1 \mathrm{C}$ shows that plain NGO $\zeta$-potentials were $-45.8 \mathrm{mV}$, and after loading of Pt complexes, NGO-Pt $\zeta$-potential was 16.1 or $21.3 \mathrm{mV}$. After NGO-Pt had been conjugated with PEI and $\mathrm{Tf}$, nanosystem $\zeta$-potential of NGO-HPIP-Pt and Tf-NGO@Pt was 44.6/37.2 mV and $40.6 / 29.2 \mathrm{mV}$, respectively. Therefore, the state of $\mathrm{NGO}$, NGO-Pt, NGO-HPIP-Pt, and Tf-NGO@Pt showed that the NGO-based nanosystem with PEI exhibited more stability in water/physiological conditions.

The chemical structure of the Tf-NGO@Pt nanosystem was characterized by FTIR, and further the UV-visible nanosystem was examined. Figure S1 shows that the characteristic NGO peaks in the FTIR spectrum at $1,727 \mathrm{~cm}^{-1}$ were absorption bands corresponding to the $\mathrm{C}=\mathrm{O}$ groups and $\mathrm{Pt}$ complexes at $1,547 \mathrm{~cm}^{-1}$. For NGO-Pt, the intensity increase for peaks at 1,500 and $1,729 \mathrm{~cm}^{-1}$ indicated that NGO-Pt was synthesized successfully. Figure S1A shows that the typical PEI-absorption peaks included $-\mathrm{CH}_{2}-$ stretching at 1,424 and $2,929 \mathrm{~cm}^{-1}$. The FTIR spectrum of NGO-Pt-PEI showed increased absorption-peak intensity at 2,959 $\mathrm{cm}^{-1}$. Representative peaks of amide I and amide II - $\mathrm{CO}-\mathrm{NH}-$ groups from Tf-NGO@Pt were 1,647 and 1,556 $\mathrm{cm}^{-1}$, respectively. Figure S1B shows that the UV-visible spectra validated that Pt complexes, PEI, and Tf were successfully incorporated on the nanoparticles from NGO spectral changes, Pt complexes, NGO-Pt, NGO-Pt-PEI, and Tf-NGO@Pt. The peak of Pt complexes indicated that Tf-NGO@Pt was successfully prepared. Drug-encapsulation efficiency of Pt complexes was $22.9 \%$, as measured by ICP-MS.

Tf-NGO@Pt stability is important in clinical application. As shown in Figure S2A, Tf-NGO@Pt size was monitored in aqueous and human plasma for 40 days. In human plasma, the average size of Tf-NGO@Pt was $45.6 \mathrm{~nm}$ and showed excellent stability. Interestingly, TfNGO@Pt size in aqueous solution remained at $39.7 \mathrm{~nm}$ for 15 days and gradually increased to $50.3 \mathrm{~nm}$ at 40 days. 
A

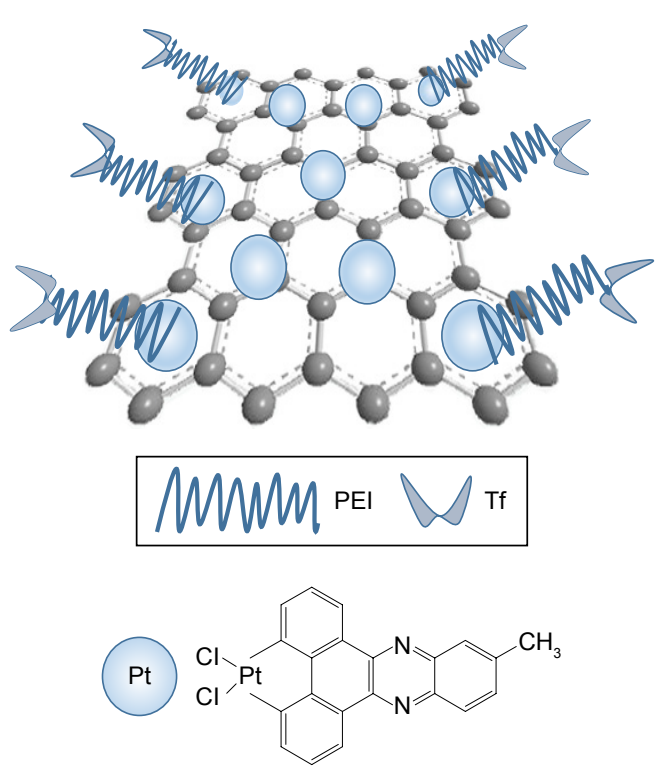

B

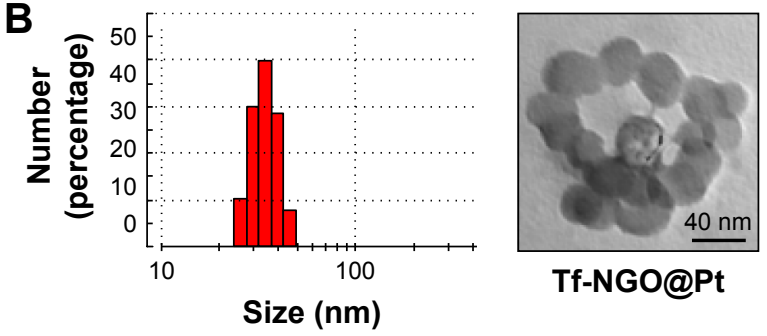

C

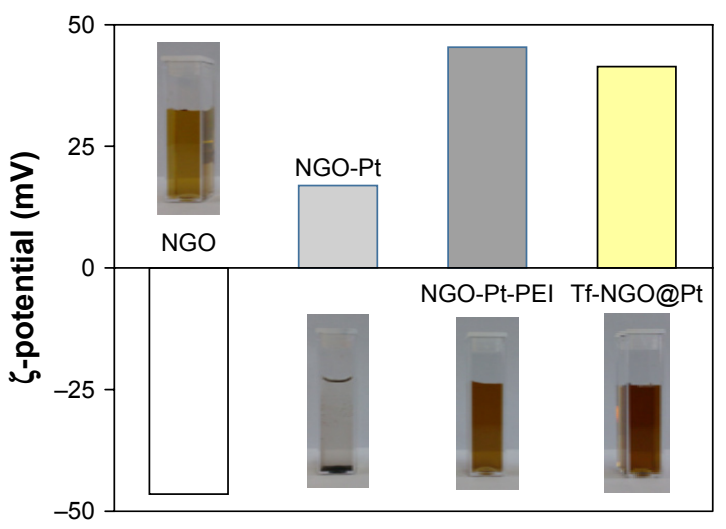

Figure I Synthesis and characterization of Tf-NGO@Pt.

Notes: (A) Structural characterization; (B) nanoparticle size and TEM results; magnification $\times 200$. (C) $\zeta$-potential and images of NGO, NGO-Pt, NGO-Pt-PEI, and Tf-NGO@Pt in aqueous solution.

Abbreviations: Tf, transferrin; NGO, nanographene oxide; TEM, transmission electron microscopy; PEl, polyethylenimine.

Overall, the Tf-NGO@Pt remained stable in conditions of aqueous solution and human plasma. In Figure S2B, changes in $\zeta$-potential for Tf-NGO@Pt in two kinds of solutions are verified over 40 days. The $\zeta$-potential for TfNGO@Pt was $40.7 \mathrm{mV}$, and only slightly reduced to average $38.4 \mathrm{mV}$ after 30 days and change to $37.6 \mathrm{mV}$ in 40 days.

\section{In vitro anticancer activity of Tf-NGO@Pt}

To determine the selectivity for Tf-NGO@Pt, MG63,HeLa, CaSki, SiHa, MCF7, A375, and HepG2 malignant cells were used as cancer-cell models, and Chem5, NIH3T3, HK2, and SV-HUVC as normal-cell models. Table 1 shows that the free nanocarrier of NGO and NGO-PEI was not cytotoxic against any cell lines at $2 \mathrm{mg} / \mathrm{mL} \mathrm{NGO}$ and NGO-PEI.Tf-NGO@Pt significantly enhanced anticancer activity for Pt and simultaneously reduced cytotoxicity toward normal cells. For example, Tf-NGO@Pt showed a 15-fold greater anticancer effect on MCF7 cells, and $\mathrm{SV}-\mathrm{HUVC}$ cells were even more cytotoxic. These results indicated that the $\mathrm{IC}_{50}$ of Tf-NGO@Pt was 6.5 $\mu \mathrm{M}$ and most sensitive to MCF7 cells. Tf-NGO@Pt considerably inhibited MG63-cell growth in a dose-dependent manner. Moreover, Figure S3 shows that Tf-NGO@Pt had higher anticancer effects than free-Pt complexes or NGO-Pt-PEI in MCF7 cells at 24 and 48 hours. Pt complexes and NGOPt-PEI expressed negligible cytotoxicity in 24 hours. Furthermore, Tf-NGO@Pt was noncytotoxic toward SV-HUVC cells ( $\left.\mathrm{IC}_{50} 95.8 \mu \mathrm{M}\right)$. In brief, Tf-NGO@Pt showed outstanding selectivity for cancer cells, ensuring its effective application with safe doses in vivo. It is possible that, the advantage of Tf-NGO@Pt could be due to Tf protein-surface decoration, which could effectively improve the selectivity of Tf-NGO@Pt between cancer and normal cells.

\section{Tf-NGO@Pt cellular uptake}

Cellular uptake assays were conducted to verify the selectivity of Tf-NGO@Pt. In brief, we quantified Tf-NGO@ Pt-uptake efficacy in MCF7 and SV-HUVC cells by the standard-curve method from internalized fluorescein isothiocyanate-loaded nanoparticles to assess Tf-NGO@ Pt selectivity. Figure $2 \mathrm{~A}$ and $\mathrm{B}$ shows that this was time dependent in the continuous process of Tf-NGO@Pt intracellular uptake in MCF7 and SV-HUVC cells, and cellular uptake of Tf-NGO@Pt in MCF7 cells was considerably higher (Figure 2A). However, Tf-NGO@Pt compared with Pt complexes showed lower fluorescence intensity in SVHUVC cells (Figure 2B). These results were expected, and indicate the feasibility of Tf-guided selectivity in Tf-NGO@ Pt between cancer cells and normal cells. 
Table I In vitro cytotoxicity of Tf-NGO@Pt (72 hours)

\begin{tabular}{|c|c|c|c|c|c|c|c|c|c|c|c|}
\hline \multirow[t]{2}{*}{ Samples } & \multicolumn{11}{|c|}{$I C_{50}(\mu M)$} \\
\hline & MG63 & HeLa & CaSki & $\mathrm{SiHa}$ & MCF7 & A375 & HepG2 & Chem5* & NIH3T3* & HK2* & SV-HUVCI* \\
\hline $\mathrm{NGO}(\mathrm{mg} / \mathrm{mL})$ & $>2.0$ & $>2.0$ & $>2.0$ & $>2.0$ & $>2.0$ & $>2.0$ & $>2.0$ & $>2.0$ & $>2.0$ & $>2.0$ & $>2.0$ \\
\hline NGO-PEI (mg/mL) & $>2.0$ & $>2.0$ & $>2.0$ & $>2.0$ & $>2.0$ & $>2.0$ & $>2.0$ & $>2.0$ & $>2.0$ & $>2.0$ & $>2.0$ \\
\hline Pt complex & 132.7 & 34.4 & 35.3 & 35.8 & 29.3 & 59.8 & 55.8 & $>150$ & $>150$ & $>150$ & 89.6 \\
\hline NGO-Pt-PEI & 126.3 & 30.6 & 31.2 & 29.6 & 10.2 & 54.7 & 50.2 & $>150$ & $>150$ & $>150$ & 92.3 \\
\hline Tf-NGO@Pt & 110.6 & 25.2 & 25.6 & 23.4 & 6.5 & 50.2 & 46.7 & $>150$ & $>150$ & $>150$ & 95.8 \\
\hline
\end{tabular}

Notes: *Chem5, NIH3T3, HK2, and SV-HUVCI are normal cells.

Abbreviations: Tf, transferrin; NGO, nanographene oxide; PEI, polyethylenimine.

The efficiency of drug retention is a crucial parameter in biological application. Therefore, we examined the fluorescence intensity of Pt complexes and Tf-NGO@Pt in cancer cells, as shown in Figure 2C. Tf-NGO@Pt compared with Pt complexes had significantly higher intensity in MCF7 cells in a time-dependent manner. However, Figure 2D shows that retention efficacy was decreased in SV-HUVC cells. These outcomes suggest that Tf-NGO@Pt-enhanced retention overwhelmed Pt complexes, which ensured effective drug accumulation in cancer cells, dramatically improving its anticancer ability in MCF7 cells.

Many studies have shown that ligand/receptor-mediated targeting is an important pathway in drug selectivity between cancer and normal cells. Therefore, we investigated Tf-NGO@ Pt selective cytotoxicity mechanisms. A Tf competing assay was conducted to verify the role of Tf/TfR-mediated targeting in the process of Tf-NGO@Pt causing cell death. Figure 3 shows that excess Tf dose dependently blocked Tf-NGO@,
A

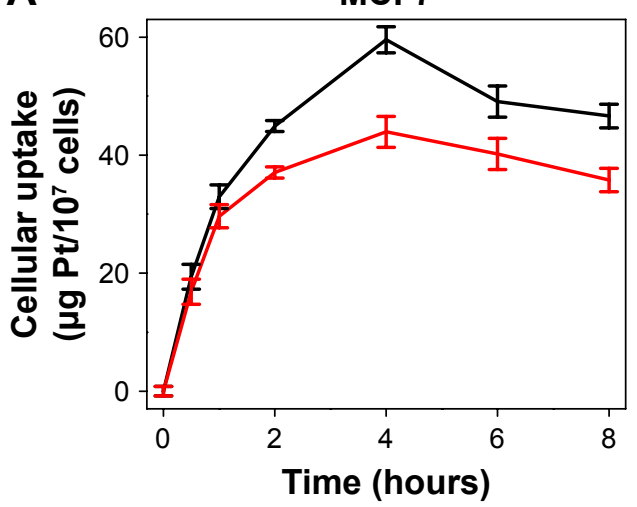

C

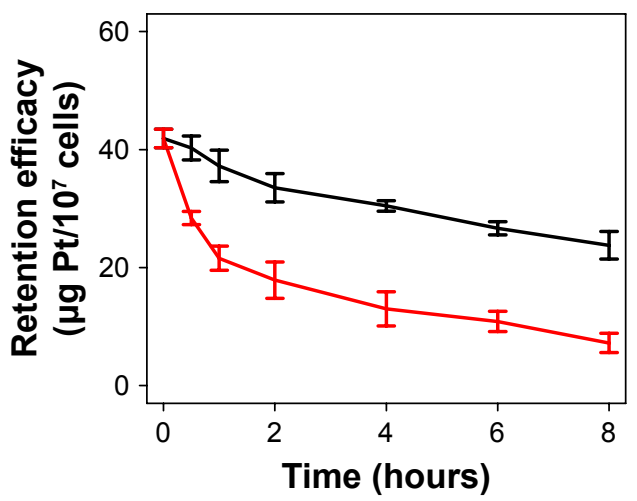

B

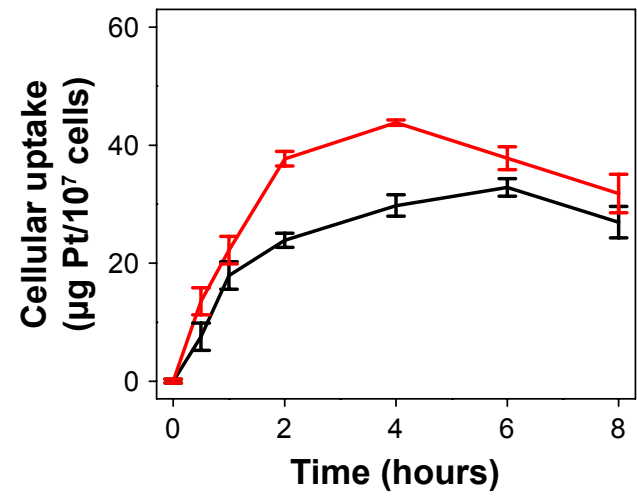

D

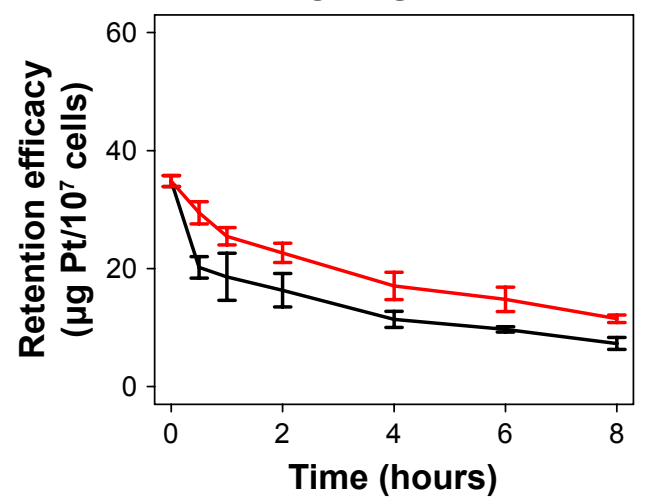

Figure 2 Selective cellular uptake and intracellular retention of Tf-NGO@Pt.

Notes: Quantitative analysis of 6-coumarin-loaded Pt complex (I2 mM) and Tf-NGO@Pt (I2 mM) by cellular uptake in MCF7 (A) and SV-HUVCI (B). Quantitative analysis of 6-coumarin-loaded Pt complex (I2 mM) and Tf-NGO@Pt (I2 mM) by retention efficacy in MCF7 (C) and SV-HUVCI (D).

Abbreviations: Tf, transferrin; NGO, nanographene oxide. 

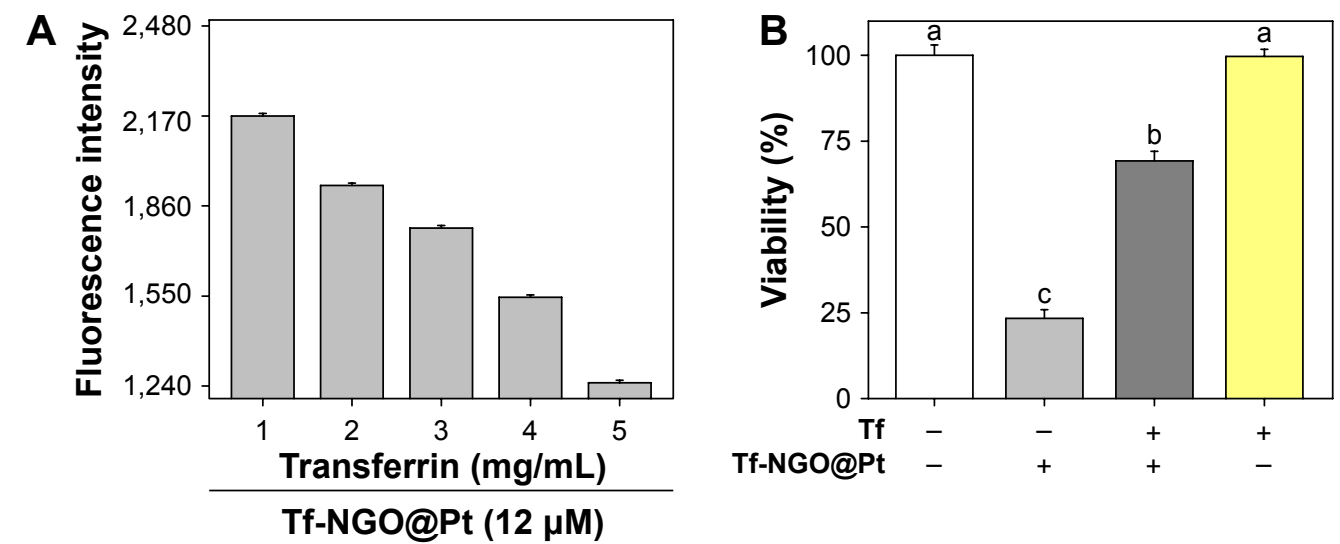

Figure 3 Cellular uptake of Tf-NGO@Pt.

Notes: (A) Cellular uptake of 6-coumarin-loaded Tf-NGO@Pt in MCF7 cells after blinding by Tf molecule; (B) viability of MCF7 cells after treatment with 6-coumarinloaded Tf-NGO@Pt (12 mM). Bars with different letters (a-c) are statistically different at the $P<0.05$ level.

Abbreviations: Tf, transferrin; NGO, nanographene oxide.

Pt uptake into MCF7 cells. Therefore, the Tf-NGO@Pt achieved upregulated cellular uptake by specifically binding to TfRs on MCF7 cells. As such, the excess Tf treatment dramatically declined Tf-NGO@Pt toxicity in MCF7 cells: $69.24 \%$ vs $23.35 \%$ from a single Tf-NGO@Pt treatment by reduced amount of internalized Tf-NGO@Pt. Taken together, these outcomes show that Tf-NGO@Pt obviously targeted cancer cells and reduced normal-cell uptake, on account of TfR-mediated endocytosis. Tf-NGO@Pt has superior selectivity uptake between cancer and normal cells.

\section{Tf-NGO@Pt intracellular localization, uptake pathways, and $\mathrm{pH}$-responsive drug release}

Figure 4 shows the process of intracellular location of Tf$\mathrm{NGO} @ \mathrm{Pt}$ in MCF7 cells. Imaging was performed to visualize the subcellular localization of Tf-NGO@Pt (Figure 4A). DAPI (blue) and LysoTracker (red) were specific probes used to label the nucleus and lysosome, respectively. Figure 4A shows that Tf-NGO@Pt localized in the lysosomes within 1 hour after being internalized, with some further gradual accumulation in the lysosome over 8 hours, shown as the high coincidence of green and red fluorescence in the cells, and shifted to the nucleus in MCF7 cells. These outcomes suggest that the lysosome is the main target of Tf-NGO@Pt after being internalized.

To examine Tf-NGO@Pt-endocytosis mechanisms, cells were pretreated with four series of endocytosis inhibitors at the initial stage. Figure 4B shows that with the combination of 2-deoxy-D-glucose and $\mathrm{NaN}_{3}$ treatments and low-temperature $\left(4^{\circ} \mathrm{C}\right)$, cellular internalization of Tf-NGO@Pt was reduced to $38.68 \%$ and $72.22 \%$, which indicates that Tf-NGO@Pt is internalized through endocytosis by energy dependent pathways to enter MCF7 cells. There were three main mechanisms of endocytosis: clathrin, caveolae/lipid raft-mediated apoptosis, and phagocytosis/macropinocytosis. ${ }^{43}$ Since MCF7 cells are not phagocytic, other pathways were investigated. The specific receptor TfR was conjugated to Tf through lipid raft-coated vesicles to bind ferric ions. ${ }^{44,45}$ Tf-NGO@Pt cellular uptake was estimated using serial treatment of sucrose, an inhibitor of clathrin-mediated endocytosis. Sucrose decreased Tf-NGO@Pt uptake to 35.37\% of control, demonstrating that clathrin-mediated endocytosis was the primary pathway. Dynamin, a GTP-binding protein, is required for receptormediated endocytosis. Dynasore, a specific inhibitor, was necessary for dynamin-mediated lipid-raft endocytosisrestricted Tf-NGO@Pt internalization to 53.9\% of control, which indicates that the dynamin-mediated pathway was the primary pattern of lipid raft-dependent endocytosis of Tf-NGO@Pt in MCF7 cells. Nystatin, a specific inhibitor for lipid raft-dependent endocytosis, reduced Tf-NGO@Pt uptake to $61.36 \%$, indicating that lipid raft-dependent endocytosis still caused Tf-NGO@Pt endocytosis. On the whole, Tf-NGO@Pt cellular uptake in MCF7 cells involved clathrin-mediated endocytic and dynamin-dependent lipid raft-mediated pathways.

To further identify clathrin-mediated endocytosis as the primary endocytic pathway, the cellular viability of MCF7 cells was determined after treatment with sucrose and Tf-NGO@Pt, as shown in Figure 4C. Pretreatment with sucrose significantly reduced Tf-NGO@Pt-caused cytotoxicity in MCF7 cells. Therefore, clathrin mediated endocytosis was confirmed as the primary endocytic pathway. Sucrose decreased Tf-NGO@Pt uptake to 35.37\% of 
A
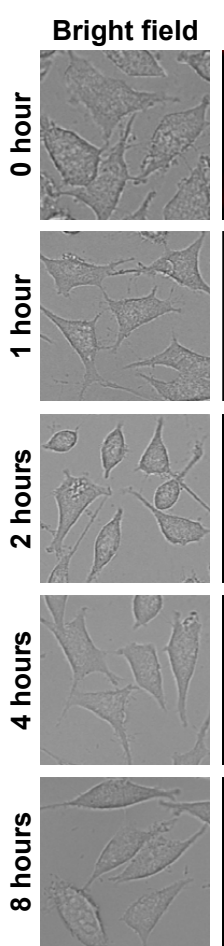
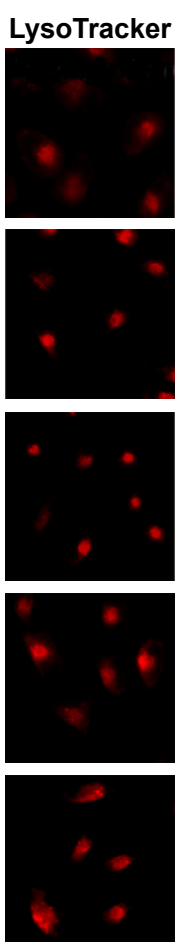
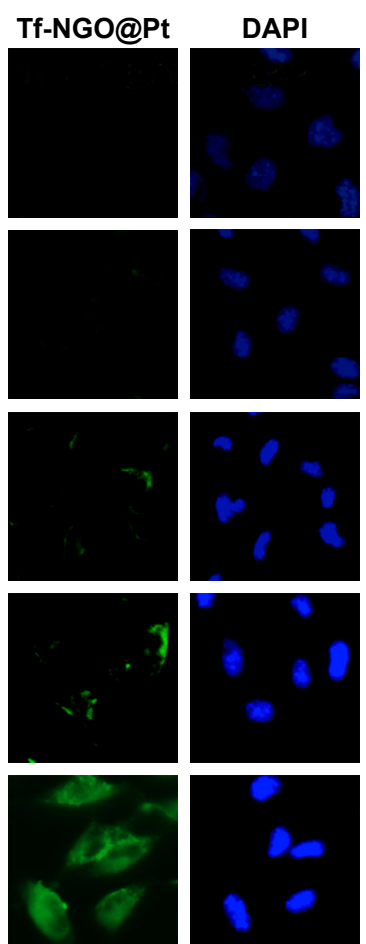
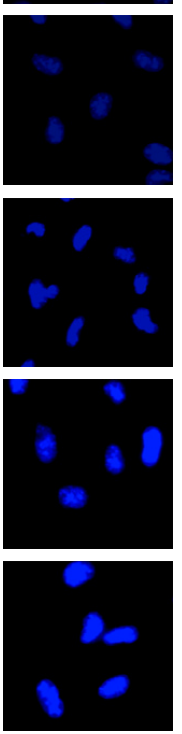

B

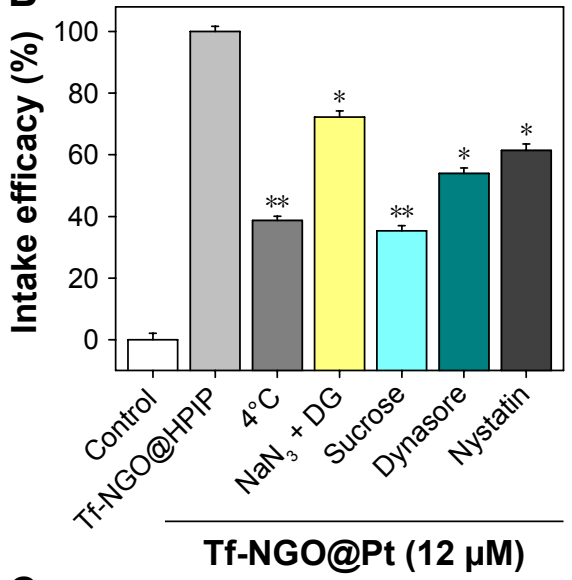

C
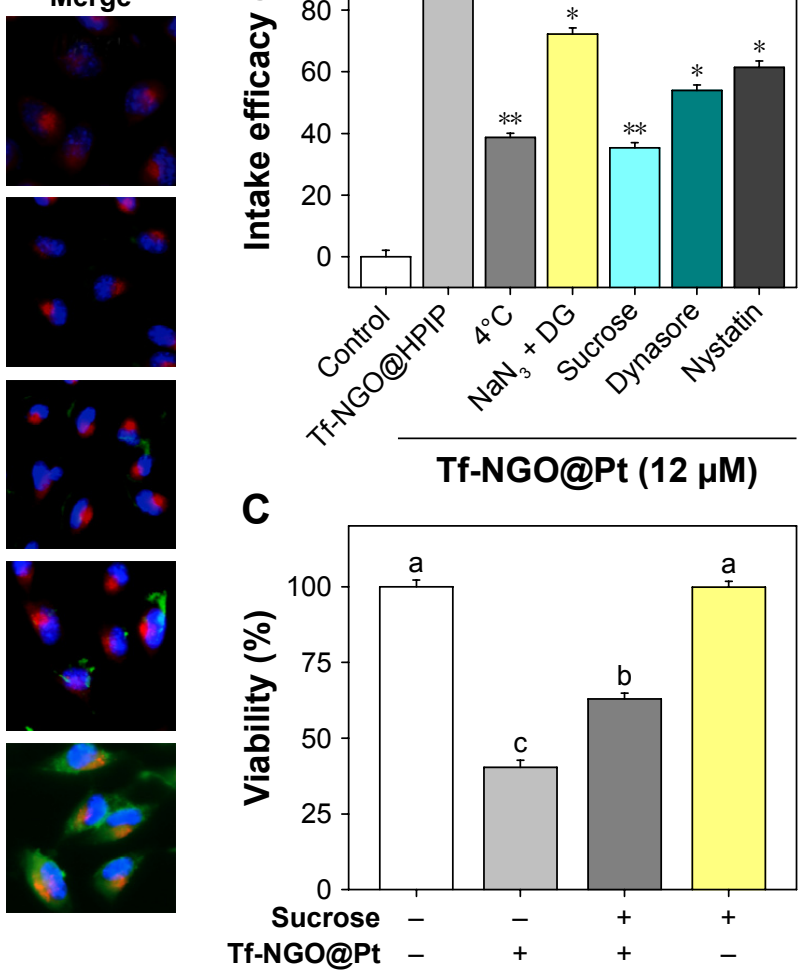

Figure 4 Intracellular localization and endocytosis mechanism of Tf-NGO@Pt.

Notes: (A) Intracellular location of Tf-NGO@Pt in MCF7 cells. Magnification $\times 200$. (B) Intracellular uptake of Tf-NGO@Pt in MCF7 cells. *P<0.05; **P<0.0I. (C) Cytotoxicity of Tf-NGO@Pt in MCF7 cells after sucrose pretreatment.

Abbreviations: Tf, transferrin; NGO, nanographene oxide; DAPI, 4',6-diamidino-2-phenylindole; DG, 2-deoxy-D-glucose.

control, confirming that clathrin-mediated endocytosis was the primary pathway. Pretreatment with dynasore significantly reduced Tf-NGO@Pt-caused cytotoxicity in MCF7 cells. These outcomes confirm dynamin-dependent lipid raft-mediated endocytosis is the primary endocytic pathway. In summary, Tf-NGO@Pt cellular uptake in MCF7 cells involves dynamin-dependent lipid raft- and clathrin-mediated endocytic pathways. For drug release,Tf-NGO@Pt powder was dispersed in PBS solution as buffer to simulate the physiological environment in cancer cells, as shown in Figure 5. Pt-complex release was $2.19 \%, 4.19 \%, 6.62 \%$, and $7.85 \%$ for $\mathrm{pH} 7.4,6.8,5.3$, and 5.3+ buffer, respectively, within 1 hour, and increased to $26.82 \%, 47 \%, 63.38 \%$, and $72.46 \%$, respectively, within 72 hours.

Generally, the NGO layer was negatively charged (Figure S2B) and conjugated with positively charged complexes. However, the complexes may compete with protons in the solution under acidic environments for interaction with the nanoparticle composition, which could allow free complexes to be released into the solution from the nanoparticle. This was verified by the nanosystem possessing $\mathrm{pH}$-responsive drug release, as indicated. In cell-lysis solution, the NGO shell was significantly dissolved, leading to rapid drug release, owing to enzymes or intracellular environments. Therefore, Tf-NGO@Pt is a bioresponsive nanomedicine within the intracellular environment.

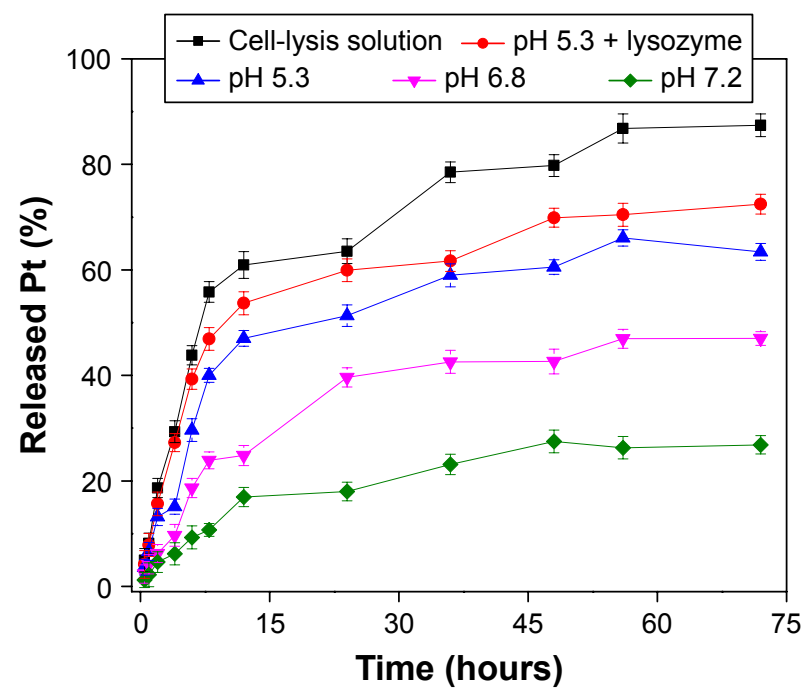

Figure 5 Drug release of complex in Tf-NGO@Pt and in vitro release of Pt complex from nanosystem in different PBS solutions.

Abbreviations: Tf, transferrin; NGO, nanographene oxide. 


\section{Selective induction of cancer-cell apoptosis}

Apoptosis has been identified as a main action mechanism in cancer treatment with metal complexes. ${ }^{30}$ This study verified Tf-NGO@Pt-action modes by flow cytometry. Figures 6 and S4 show that Pt complexes and Tf-NGO@Pt induced large-scale apoptosis in MCF7 cells (see the sub-G $\mathrm{G}_{1}$ populations in the graphs). Tf-NGO@Pt possessed a particularly higher level of apoptosis than Pt complexes. For example, MCF7 cells treated with 3, 6, and $12 \mu \mathrm{M}$ Tf-NGO@Pt increased from $0.2 \%$ (control) to $17 \%, 31.7 \%$, and $46.4 \%$, significantly larger than those of $\mathrm{Pt}$ complexes $(7.5 \%$, $10.1 \%$, and $16.3 \%$, respectively) at equivalent concentrations. In contrast, Pt complexes and Tf-NGO@Pt had negligible apoptosis in SV-HUVC cells. Furthermore, as shown in Figure 7, TUNEL-assay results showed that Tf-NGO@ Pt caused a dose-dependent increase in DNA fragmentation (green fluorescence) and nucleus condensation (blue fluorescence) in MCF7 cells. These results demonstrate that cell death induced by Tf-NGO@Pt nanoparticles was caused mainly by apoptosis. In sum, Tf-NGO@Pt was verified to induce MCF7 apoptosis, but showed lower effects on normal cells.

\section{Tf-NGO@Pt triggers ROS overproduction in cancer cells}

ROS plays an important role in cell apoptosis, which involves hydrogen peroxide, superoxide, and hydroxyl radicals. High ROS levels induce macromolecular damage and ROS-mediated apoptosis. To investigate intracellular ROS induced by Tf-NGO@Pt, we examined intracellular ROS levels by measuring the DHE-fluorescence intensity. Figure 8A and B shows that Tf-NGO@Pt overwhelmed Pt complexes by triggering ROS overproduction in MCF7 cells after 5 minutes of treatment, then reached $232.78 \%$ at 50 minutes, with a gradual reduction to $147.97 \%$. In contrast, Pt complexes and Tf-NGO@Pt caused lower ROS levels in SV-HUVC cells after 2 hours. Figure 8C shows that Tf-NGO@Pt induced strong fluorescence intensity in MCF7 cells but only slight fluorescence in SV-HUVC cells, which verifies selective ROS induction by Tf-NGO@Pt. In contrast, Tf-NGO@Pt caused lower ROS levels in SV-HUVC cells compared to Pt after 2-hour incubation, which can be attributed to limited Tf-NGO@Pt uptake, as discussed earlier. Therefore,Tf-NGO@Pt exerted significantly higher anticancer effects in MCF7 cells via ROS-activated apoptosis.

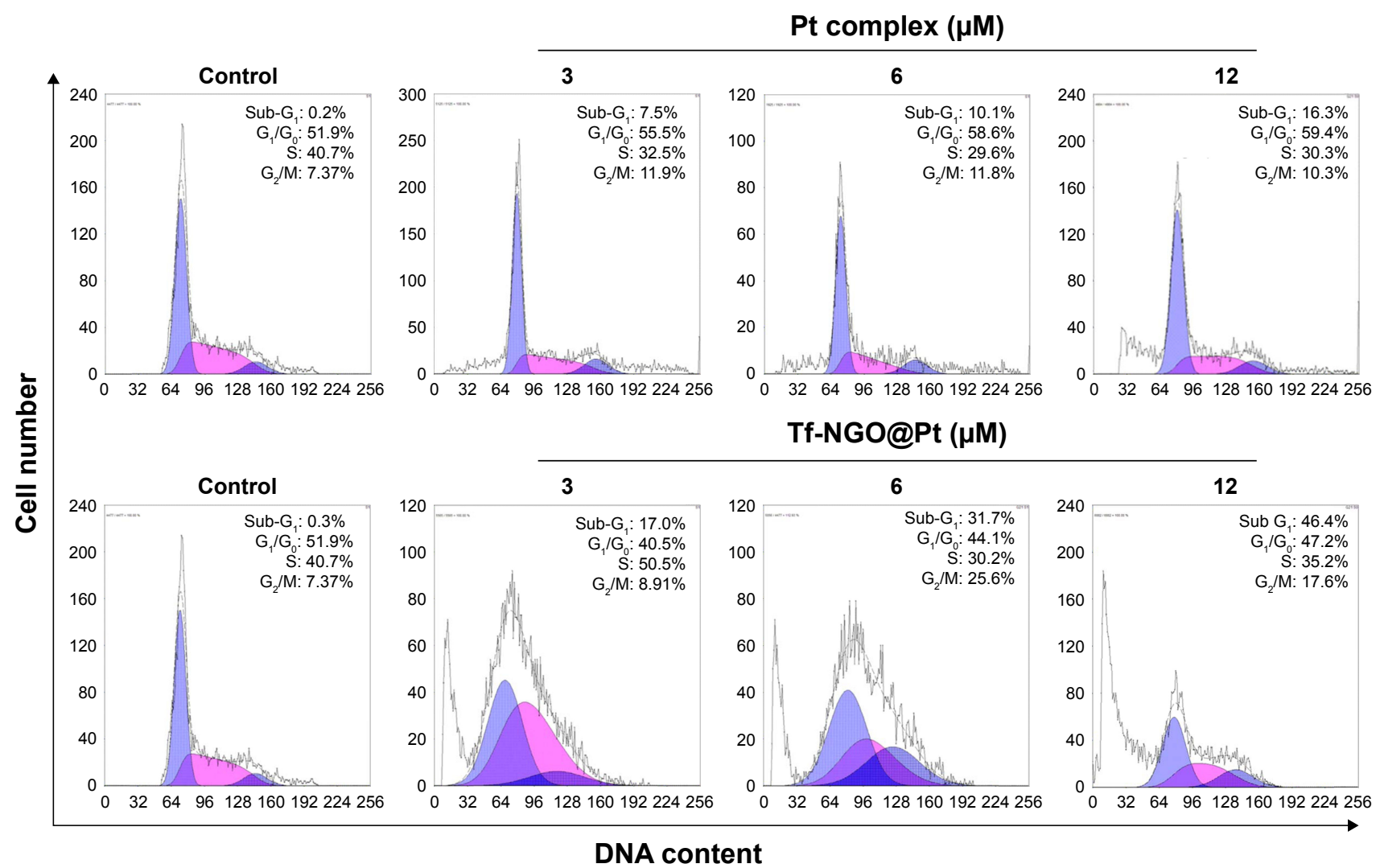

Figure 6 Flow-cytometry analysis of MCF7 cells after incubation with Pt complex and Tf-NGO@Pt.

Abbreviations: Tf, transferrin; NGO, nanographene oxide. 


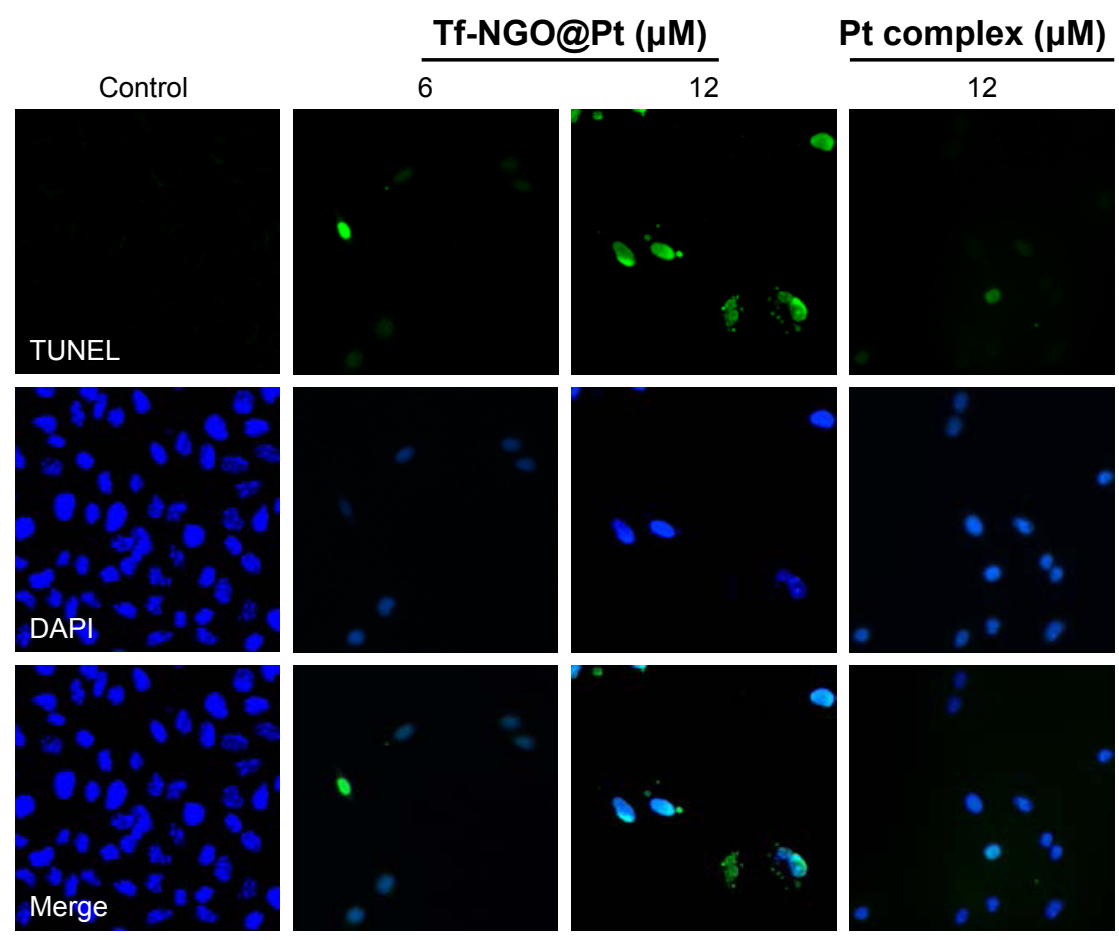

Figure 7 Representative images of DNA fragmentation and nuclear condensation.

Note: Fragmentation and condensation caused by 24-hour treatment of Pt complex (I2 $\mu \mathrm{M})$ and Tf-NGO@Pt (6 and I $2 \mu \mathrm{M})$, as detected by TUNEL assay and DAPI staining. Magnification $\times 200$.

Abbreviations: Tf, transferrin; NGO, nanographene oxide; TUNEL, terminal deoxynucleotidyl transferase dUTP nick end labeling; DAPI, 4',6-diamidino-2-phenylindole.
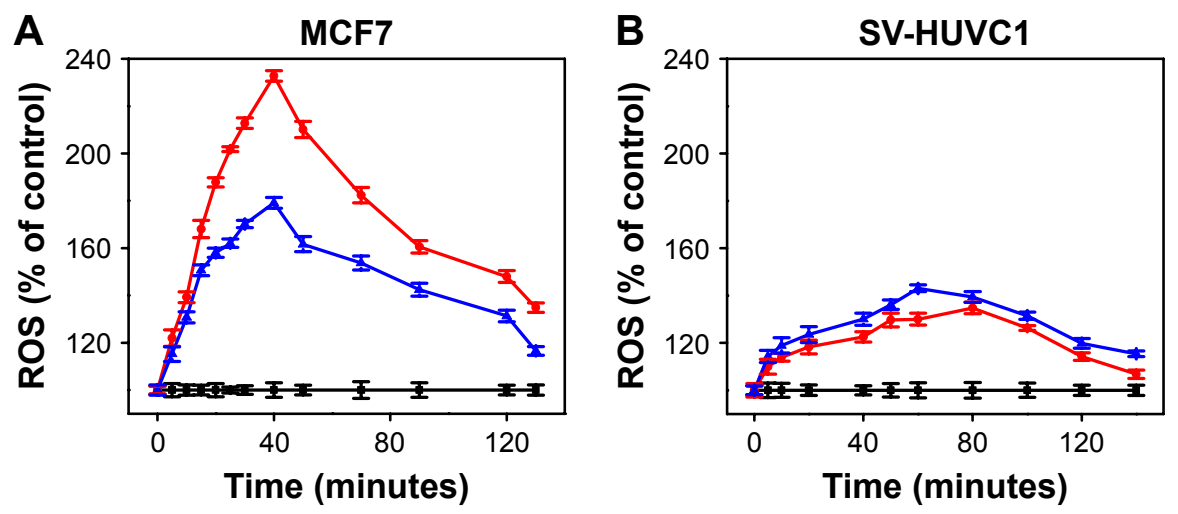

C
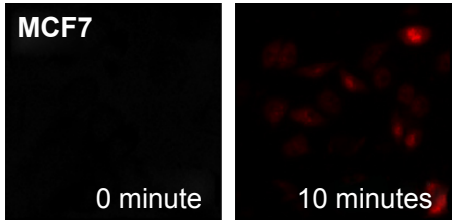

$\rightarrow-$ Control $\rightarrow$ Tf-NGO@Pt $\leftarrow$ Pt complex
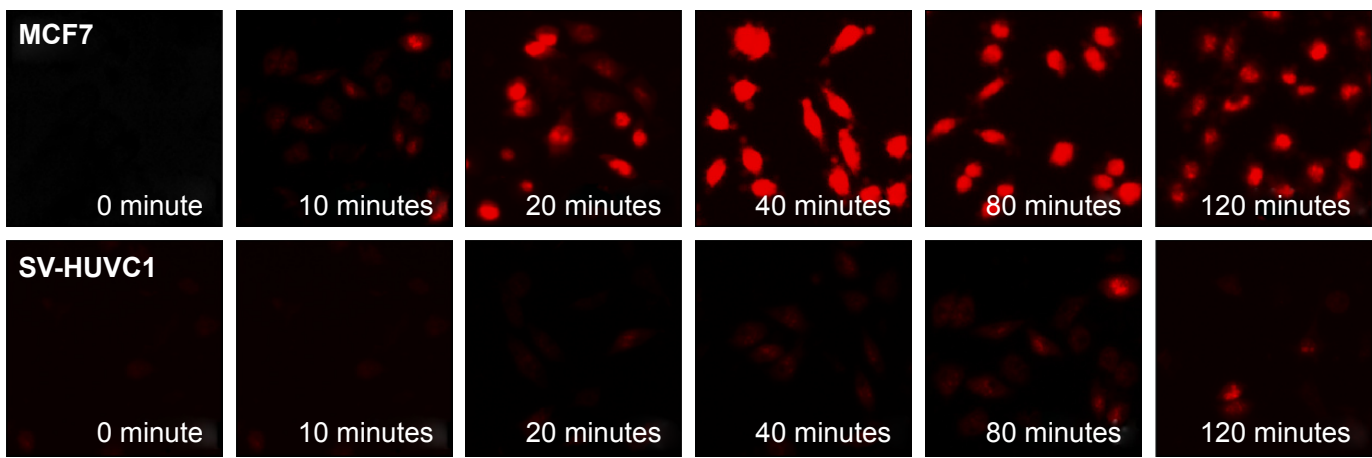

Figure 8 Changes of ROS generation induced by Tf-NGO@Pt.

Notes: (A) MCF7 cells were treated with Pt complex and Tf-NGO@Pt for 120 minutes; (B) SV-HUVCl cells were exposed to different concentrations of Pt complex and Tf-NGO@Pt for 120 minutes; (C) MCF7 and SV-HUVCI cells were dyed by $10 \mu$ M DHE at 0, 10, 20, 40, 80, I20 minutes. Magnification $\times 200$.

Abbreviations: ROS, reactive oxygen species; Tf, transferrin; NGO, nanographene oxide; DHE, dihydroethidium. 


\section{Tf-NGO@Pt penetration ability and inhibitory effects on MCF7 spheroids}

Less sensitivity to drugs has been reported for cancer cells in tumors than in cultured cells. ${ }^{42}$ Recently, tumor-spheroid cellculture systems have received increasing interest, because they can restore in vivo environments. ${ }^{46}$ In particular, tumor spheroids with diameters up to $200 \mathrm{~mm}$ sufficiently reflect in vivo like cell-cell and cell-matrix interactions. ${ }^{47}$ This study successfully cultivated MCF7 spheroids following an in vitro formation process (Figure 9A).

To examine the penetration ability of different Tf-NGO@Pt nanosystems, MCF7 spheroids were treated with fluorescein isothiocyanate-labeled nanoparticles for 12 hours. The resultant tumor spheroids were scanned at different layers from the top of the spheroid to the middle using confocal laserscanning fluorescent microscopy, as shown in Figure 9C. Tumor spheroids treated with the nanoparticles displayed varied fluorescence intensity. Among them, Tf-NGO@Pt showed excellent advantages in penetrating ability of the MCF7 spheroids, reaching the core.

Figure 9B shows MCF7 spheroid-volume ratios measured after treatment with free complexes and Tf-NGO@Pt nanoparticles. Tf-NGO@Pt significantly inhibited growth of the MCF7 spheroids. For example, Tf-NGO@Pt reduced MCF7 spheroid-volume ratios by $71 \%$ of control after 120 hours, significantly more effective than free-Pt complexes (31.4\%). Therefore, although formation of a protein corona was observed, Tf-NGO@Pt showed excellent advantages in penetration ability and inhibitory effects for future clinical use.

\section{Tf-NGO@Pt suppresses MCF7-cell migration and invasion in vitro}

Cell migration and invasion are also pivotal for tumor growth. ${ }^{48}$ To investigate the antimetastasis and invasion ability of Tf-NGO@Pt, we conducted wound-healing and transwell assays, as shown in Figure 10A and D. Migration and invasion of MCF7 cells were effectively suppressed by free complexes, but Tf-NGO@Pt exhibited significantly higher inhibition than the free complexes. In particular, Tf-NGO@Pt exhibited higher activity than the free complexes. Figures 9C and 10B show the migratory and invaded ratios. These results further confirm that functionalized Tf-NGO@Pt nanosystems possess remarkable anticancer activity and application potential in cancer therapy.
A

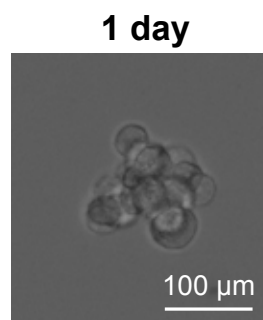

B

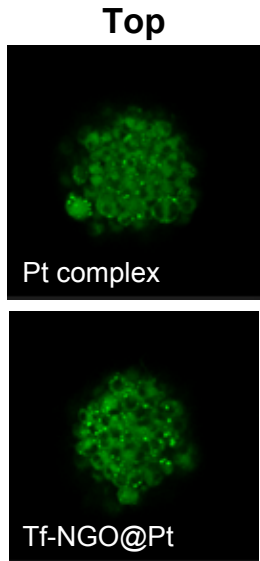

5 day

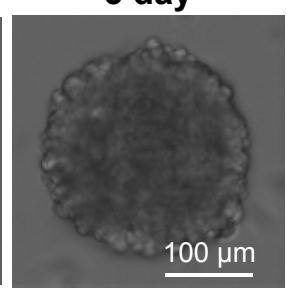
$-10 \mu \mathrm{m}$
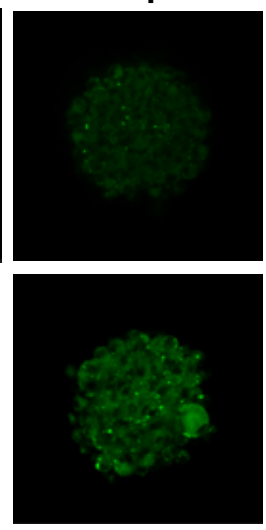

C

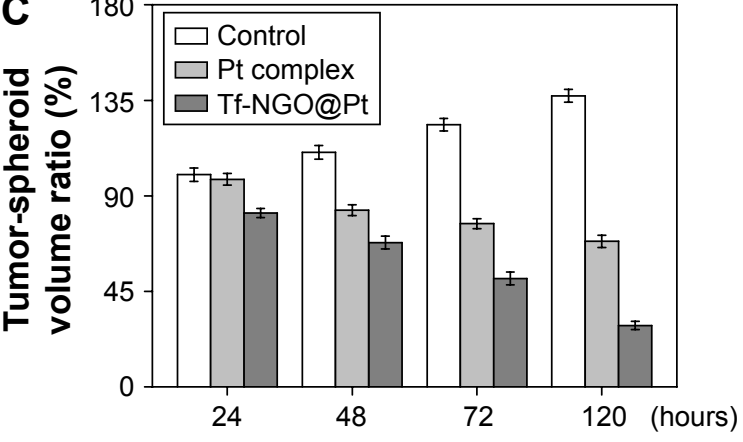

$-20 \mu \mathrm{m}$

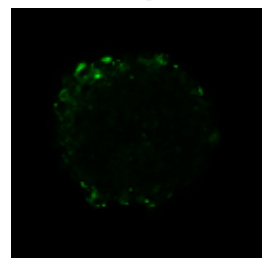

$-30 \mu \mathrm{m}$

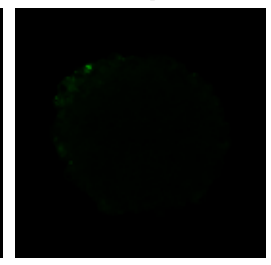

$-50 \mu \mathrm{m}$

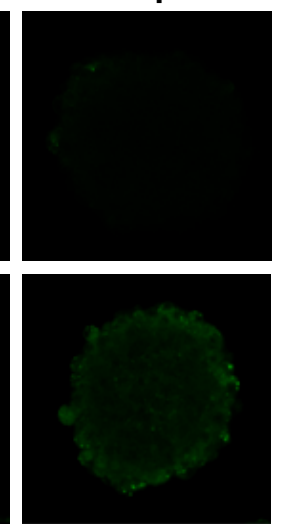

Figure 9 Penetration ability and inhibitory effect toward MCF7 spheroids.

Notes: (A) The formation process of MCF7 tumor spheroids. (B) Inhibitory effect of Pt complex and Tf-NGO@Pt toward MCF7 spheroids. Magnification $\times 200$. Values presented as mean \pm SD of triplicate results. (C) Penetration ability of Tf-NGO@Pt to MCF7 tumor spheroids. The spheroids were treated with coumarin-6-labeled nanoparticles for 12 hours. Drug concentration was $60 \mathrm{mM}$.

Abbreviations: Tf, transferrin; NGO, nanographene oxide. 
A
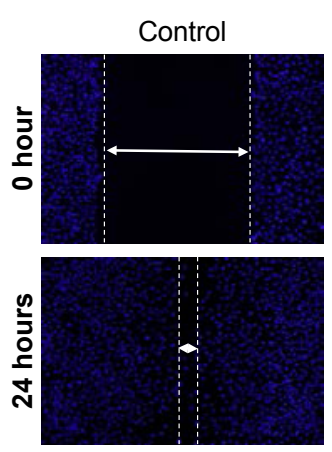

B

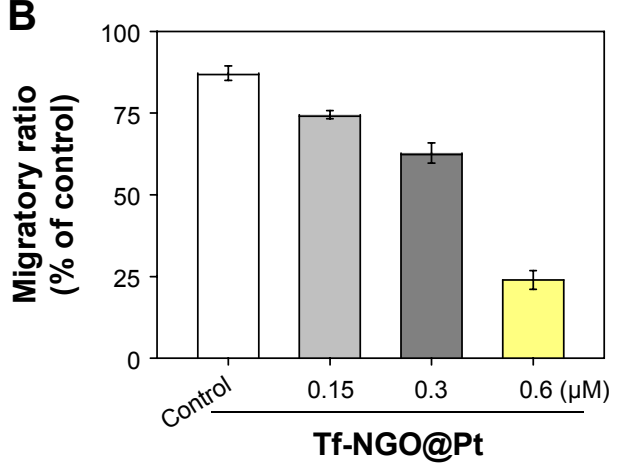

D
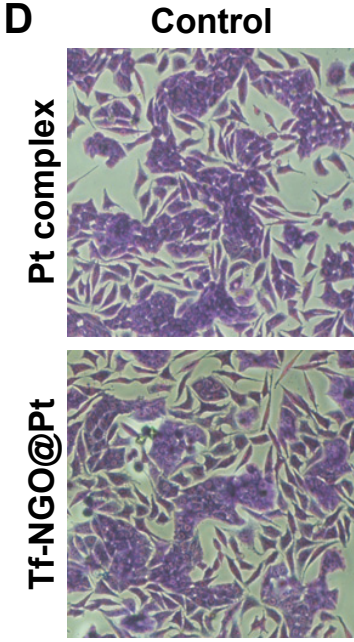

0.15

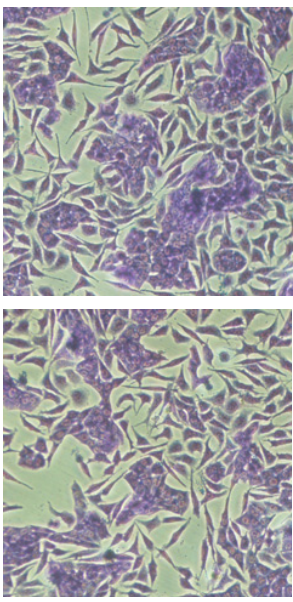

Tf-NGO@Pt ( $\mu M)$
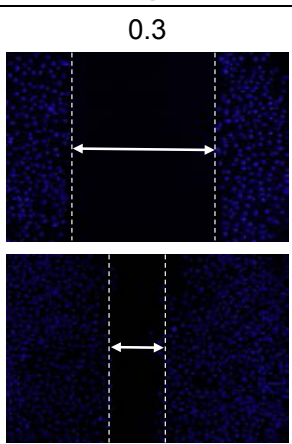

C

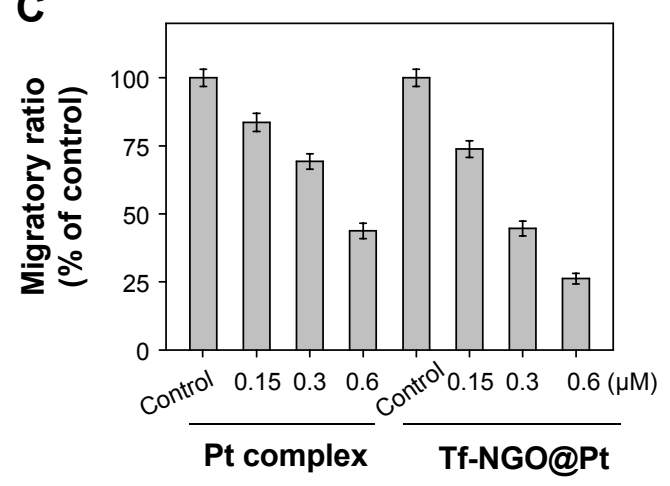

0.3

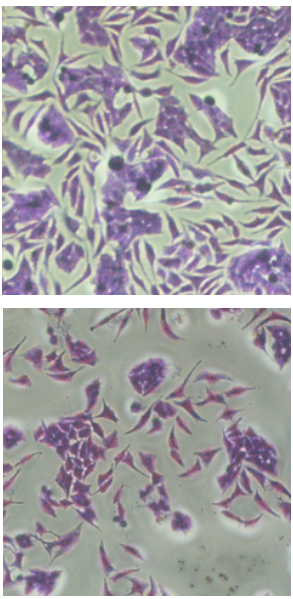

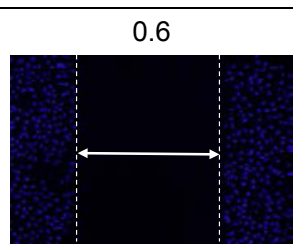

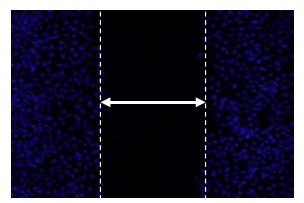

$0.6(\mu \mathrm{M})$

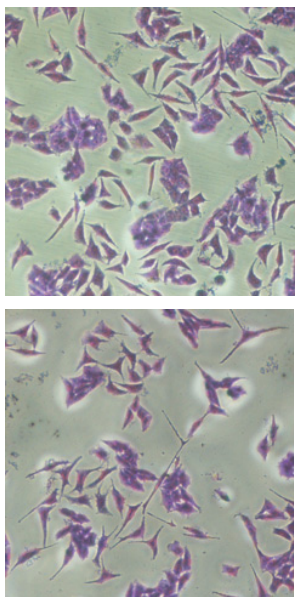

Figure 10 Wound-healing assay of Pt complex and Tf-NGO@Pt on MCF7 cells (4×I05 cells/L).

Notes: (A) Migratory assay in MCF7 cells induced by Pt complex and Tf-NGO@Pt. (B) Migratory ratio of Tf-NGO@Pt. Quantitative data were analyzed by manual counting (\% of control). (C) Invasion assay in MCF7 cells induced by Pt complex and Tf-NGO@Pt. (D) Invasion ratio of Pt complex and Tf-NGO@Pt. Magnification ×200. Quantitative data were analyzed by manual counting (\% of control). Values expressed as mean \pm SD of triplicate results.

Abbreviations: Tf, transferrin; NGO, nanographene oxide.

\section{Conclusion}

In summary, we have design and synthesized a cancertargeted Tf-NGO@Pt nanosystem, in order to overcome the poor solution of metal complexes and to enhance their anticancer efficacy. The Tf-NGO@Pt nanosystem through transferrin decorated effectively enhances the cellular uptake of drugs with increased discrimination between cancer and normal cells. The efficacy and safety index of Tf-NGO@Pt were greater than that of the free-metal complexes. This effect was closely related to the enhancement of cellular uptake and cellular drug retention. Moreover, Tf-NGO@Pt nanoparticles have shown excellent advantages in penetration ability and inhibitory effect toward MCF7 spheroids compared to free complexes. This study highlights the usefulness of cancer-targeted nanoparticles for delivery of anticancer metal complexes to achieve enhanced anticancer efficacy and to expand the usefulness of metal complexes. 


\section{Disclosure}

The authors report no conflicts of interest in this work.

\section{References}

1. Siegel RL, Miller KD, Jemal A. Cancer statistics, 2015. CA Cancer J Clin. 2015;65(1):5-29.

2. Xie L, Luo Z, Zhao Z, Chen T. Anticancer and antiangiogenic iron(II) complexes that target thioredoxin reductase to trigger cancer cell apoptosis. J Med Chem. 2017;60(1):202-214.

3. Zhang W, Guo Z, Huang D, Liu Z, Guo X, Zhong H. Synergistic effect of chemo-photothermal therapy using PEGylated graphene oxide. Biomaterials. 2011;32(33):8555-8561.

4. Zhang L, Lu Z, Zhao Q, Huang J, Shen H, Zhang Z. Enhanced chemotherapy efficacy by sequential delivery of siRNA and anticancer drugs using PEI-grafted graphene oxide. Small. 2011;7(4):460-464.

5. Zhang XF, Gurunathan S. Biofabrication of a novel biomoleculeassisted reduced graphene oxide: an excellent biocompatible nanomaterial. Int J Nanomedicine. 2016;11:6635-6649.

6. Yang K, Feng LZ, Shi XZ, Liu Z. Nano-graphene in biomedicine: theranostic applications. Chem Soc Rev. 2013;42(2):530-547.

7. Wong BS, Yoong SL, Jagusiak A, et al. Carbon nanotubes for delivery of small molecule drugs. Adv Drug Deliv Rev. 2013;65(15):1964-2015.

8. Heo DN, Yang DH, Moon HJ, et al. Gold nanoparticles surfacefunctionalized with paclitaxel drug and biotin receptor as theranostic agents for cancer therapy. Biomaterials. 2012;33(3):856-866.

9. Yang HW, Huang CY, Lin CW, et al. Gadolinium-functionalized nanographene oxide for combined drug and microRNA delivery and magnetic resonance imaging. Biomaterials. 2014;35(24):6534-6542.

10. Chung C, Kim YK, Shin D, Ryoo SR, Hong BH, Min DH. Biomedical applications of graphene and graphene oxide. Acc Chem Res. 2013; 46(10):2211-2224.

11. Sun TM, Zhang YS, Pang B, Hyun DC, Yang MX, Xia YN. Engineered nanoparticles for drug delivery in cancer therapy. Angew Chem Int Ed Engl. 2014;53(46):12320-12364.

12. He LZ, Chen TF, You YY, et al. A cancer-targeted nanosystem for delivery of gold(III) complexes: enhanced selectivity and apoptosisinducing efficacy of a gold(III) porphyrin complex. Angew Chem Int Ed Engl. 2014;53(46):12532-12536.

13. Zhong YA, Meng FH, Deng C, Zhong ZY. Ligand-directed active tumor-targeting polymeric nanoparticles for cancer chemotherapy. Biomacromolecules. 2014;15(6):1955-1969.

14. Srinivasarao M, Galliford CV, Low PS. Principles in the design of ligand-targeted cancer therapeutics and imaging agents. Nat Rev Drug Discov. 2015;14(3):203-219.

15. Bi Y, Hao F, Yan GD, Teng LS, Lee RJ, Xie J. Actively targeted nanoparticles for drug delivery to tumor. Curr Drug Metab. 2016;17(8): 763-782.

16. Akhtar MJ, Ahamed M, Alhadlaq HA, Alrokayan SA, Kumar S. Targeted anticancer therapy: overexpressed receptors and nanotechnology. Clin Chim Acta. 2014;436:78-92.

17. Kang CS, Ren SY, Sun X, Chong HS. Theranostic polyaminocarboxylatecyanine-transferrin conjugate for anticancer therapy and near-infrared optical imaging. ChemMedChem. 2016;11(19):2188-2193.

18. Tortorella S, Karagiannis TC. Transferrin receptor-mediated endocytosis: a useful target for cancer therapy. J Membr Biol. 2014;247(4): 291-307.

19. Yang Y, Zhang XM, Wang XF, et al. Enhanced delivery of artemisinin and its analogues to cancer cells by their adducts with human serum transferrin. Int J Pharm. 2014;467(1-2):113-122.

20. Li YH, Guo M, Lin ZF, et al. Polyethylenimine-functionalized silver nanoparticle-based co-delivery of paclitaxel to induce HepG2 cell apoptosis. Int J Nanomed. 2016;11:6693-6702.

21. Gorrini C, Harris IS, Mak TW. Modulation of oxidative stress as an anticancer strategy. Nat Rev Drug Discov. 2013;12(12):931-947.

22. Ichikawa Y, Ghanefar M, Bayeva M, et al. Cardiotoxicity of doxorubicin is mediated through mitochondrial iron accumulation. J Clin Invest. 2014;124(2):617-630.
23. Xiong XX, Liu JM, Qiu XY, Pan F, Yu SB, Chen XQ. Piperlongumine induces apoptotic and autophagic death of the primary myeloid leukemia cells from patients via activation of ROS-p38/JNK pathways. Acta Pharmacol Sin. 2015;36(3):362-374.

24. Kello M, Drutovic D, Chripkova M, et al. ROS-dependent antiproliferative effect of brassinin derivative homobrassinin in human colorectal cancer Caco2 cells. Molecules. 2014;19(8):10877-10897.

25. Hummers WS, Offeman RE. Preparation of graphitic oxide. J Am Chem Soc. 1958;80(6):1339.

26. Uhl FM, Wilkie CA. Preparation of nanocomposites from styrene and modified graphite oxides. Polym Degrad Stab. 2004;84(2):215-226.

27. Marcano DC, Kosynkin DV, Berlin JM, et al. Improved synthesis of graphene oxide. ACS Nano. 2010;4(8):4806-4814.

28. Liu Q, Cheng M, Wang J, Jiang G. Graphene oxide nanoribbons: improved synthesis and application in MALDI mass spectrometry. Chemistry. 2015;21(14):5594-5599.

29. Liu Z, Fan AC, Rakhra K, et al. Supramolecular stacking of doxorubicin on carbon nanotubes for in vivo cancer therapy. Angew Chem Int Ed Engl. 2009;48(41):7668-7672.

30. Xie Q, Lan GQ, Zhou YL, et al. Strategy to enhance the anticancer efficacy of X-ray radiotherapy in melanoma cells by platinum complexes, the role of ROS-mediated signaling pathways. Cancer Lett. 2014;354(1): 58-67.

31. Li Y, Li X, Zheng W, Fan C, Zhang Y, Chen T. Functionalized selenium nanoparticles with nephroprotective activity, the important roles of ROS-mediated signaling pathways. J Mater Chem B Mater Biol Med. 2013;1(46):6365-6372.

32. Liu T, Lai LH, Song ZH, Chen TF. A sequentially triggered nanosystem for precise drug delivery and simultaneous inhibition of cancer growth, migration, and invasion. Adv Funct Mater. 2016;26(43):7775-7790.

33. Huang YY, Huang W, Chan L, Zhou BW, Chen TF. A multifunctional DNA origami as carrier of metal complexes to achieve enhanced tumoral delivery and nullified systemic toxicity. Biomaterials. 2016; 103:183-196.

34. Lai H, Zhang X, Feng P, Xie L, Chen J, Chen T. Enhancement of antiangiogenic efficacy of iron(II) complex by selenium substitution. Chem Asian J. 2017;12(9):982-987.

35. Liu W, Li XL, Wong YS, et al. Selenium nanoparticles as a carrier of 5-fluorouracil to achieve anticancer synergism. ACS Nano. 2012;6(8): 6578-6591.

36. Song YM, Wu Q, Yang PJ, Luan NN, Wang LF, Liu YM. DNA binding and cleavage activity of Ni(II) complex with all-trans retinoic acid. J Inorg Biochem. 2006;100(10):1685-1691.

37. Yang F, Tang QM, Zhong XY, et al. Surface decoration by Spirulina polysaccharide enhances the cellular uptake and anticancer efficacy of selenium nanoparticles. Int J Nanomedicine. 2012;7:835-844.

38. Chen TF, Mei WJ, Wong YS, et al. Chiral ruthenium polypyridyl complexes as mitochondria-targeted apoptosis inducers. MedChem Comm. 2010;1(1):73-75.

39. Chen T, Wong YS. Selenocystine induces caspase-independent apoptosis in MCF-7 human breast carcinoma cells with involvement of p53 phosphorylation and reactive oxygen species generation. Int J Biochem Cell Biol. 2009;41(3):666-676.

40. Zheng SY, Li XL, Zhang YB, et al. PEG-nanolized ultrasmall selenium nanoparticles overcome drug resistance in hepatocellular carcinoma HepG2 cells through induction of mitochondria dysfunction. Int $J$ Nanomedicine. 2012;7:3939-3949.

41. Li LL, Cao WQ, Zheng WJ, Fan CD, Chen TF. Ruthenium complexes containing 2,6-bis(benzimidazolyl)pyridine derivatives induce cancer cell apoptosis by triggering DNA damage-mediated p53 phosphorylation. Dalton Trans. 2012;41(41):12766-12772.

42. Zhang L, Zhang Y, Huang PY, Xu F, Peng PJ, Guan ZZ. Phase II clinical study of gemcitabine in the treatment of patients with advanced nasopharyngeal carcinoma after the failure of platinum-based chemotherapy. Cancer Chemother Pharmacol. 2008;61(1):33-38.

43. Wang L, Liu Y, Li W, et al. Selective targeting of gold nanorods at the mitochondria of cancer cells: implications for cancer therapy. Nano Lett. 2011;11(2):772-780. 
44. Grant BD, Donaldson JG. Pathways and mechanisms of endocytic recycling. Nat Rev Mol Cell Biol. 2009;10(9):597-608.

45. Lakadamyali M, Rust MJ, Zhuang X. Ligands for clathrin-mediated endocytosis are differentially sorted into distinct populations of early endosomes. Cell. 2006;124(5):997-1009.

46. Yamamoto N, Renfrew AK, Kim BJ, Bryce NS, Hambley TW. Dual targeting of hypoxic and acidic tumor environments with a cobalt(III) chaperone complex. J Med Chem. 2012;55(24):11013-11021.
47. Li XY, Zhao Y, Sun MG, et al. Multifunctional liposomes loaded with paclitaxel and artemether for treatment of invasive brain glioma. Biomaterials. 2014;35(21):5591-5604.

48. Miyo M, Yamamoto H, Konno M, et al. Tumour-suppressive function of SIRT4 in human colorectal cancer. Br J Cancer. 2015;113(3): 492-499. 


\section{Supplementary materials}

A

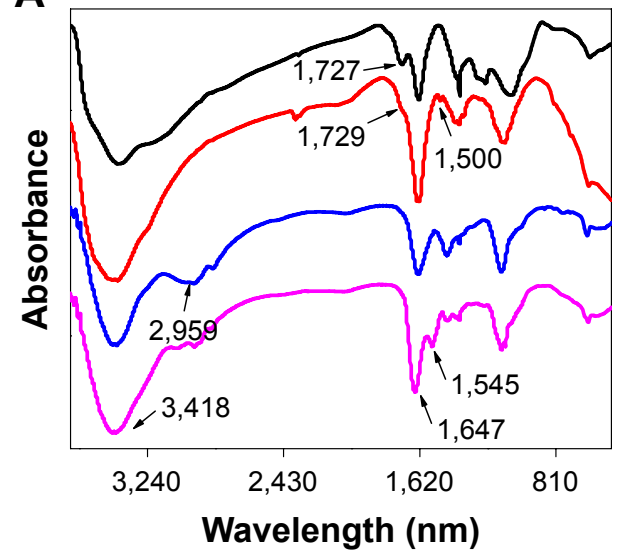

\begin{tabular}{ll|}
\hline - NGO & -NGO-Pt \\
- NGO-Pt-PEI & -Tf-NGO@Pt \\
\hline
\end{tabular}
B

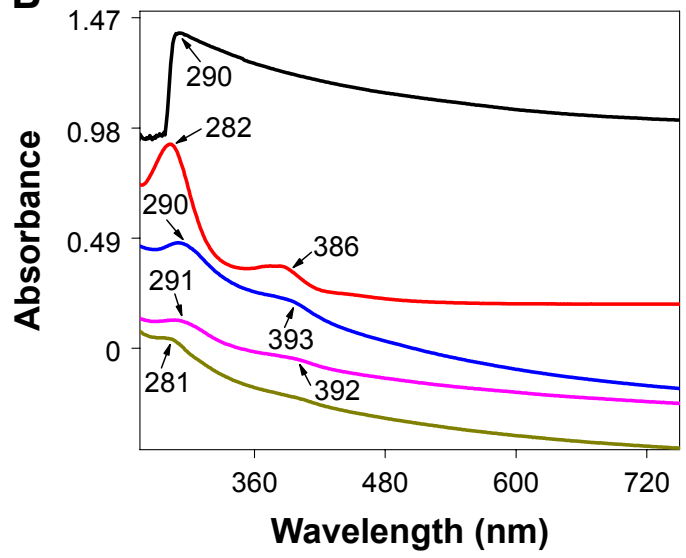

-NGO -Pt complex

- NGO-Pt -NGO-Pt-PEI

-Tf-NGO@Pt

Figure SI Structural characterization of Tf-NGO@Pt complex.

Notes: (A) Fourier-transform infrared spectra of Tf-NGO@Pt; (B) ultraviolet-visible spectra of Tf-NGO@Pt.

Abbreviations: Tf, transferrin; NGO, nanographene oxide.
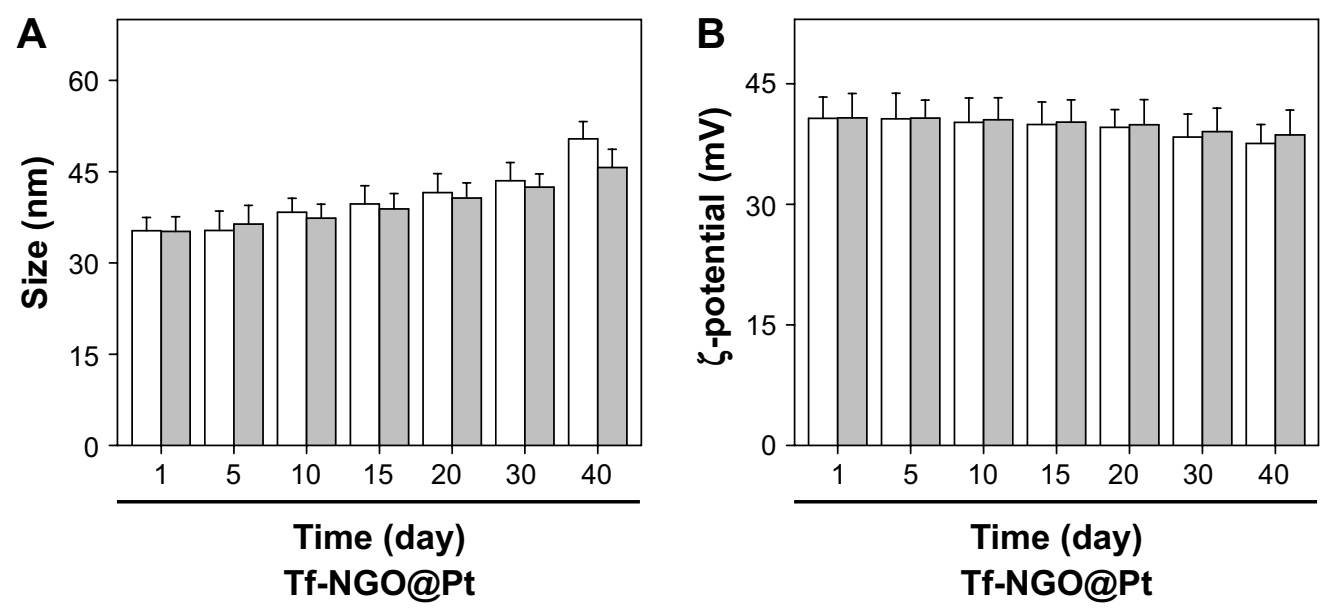

$\square$ Water $\square$ Human serum

Figure S2 Structural characterization of Tf-NGO@Pt complex.

Notes: (A) Time course of size distribution of Tf-NGO@Pt (2 mg/mL); (B) time course of $\zeta$-potential of Tf-NGO@Pt (2 mg/mL).

Abbreviations: Tf, transferrin; NGO, nanographene oxide. 


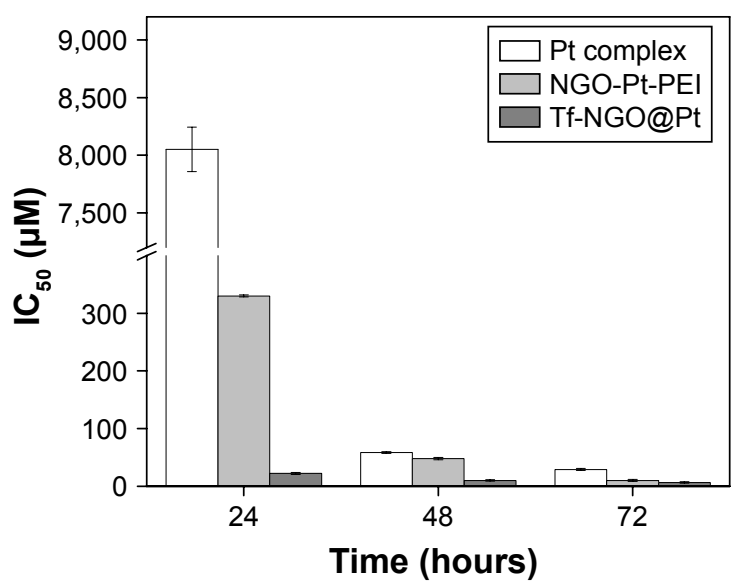

Figure S3 Cytotoxicity of Pt complex, NGO-Pt-PEI, and Tf-NGO@Pt in MCF7 cells at 24, 48, and 72 hours. Abbreviations: Tf, transferrin; NGO, nanographene oxide.

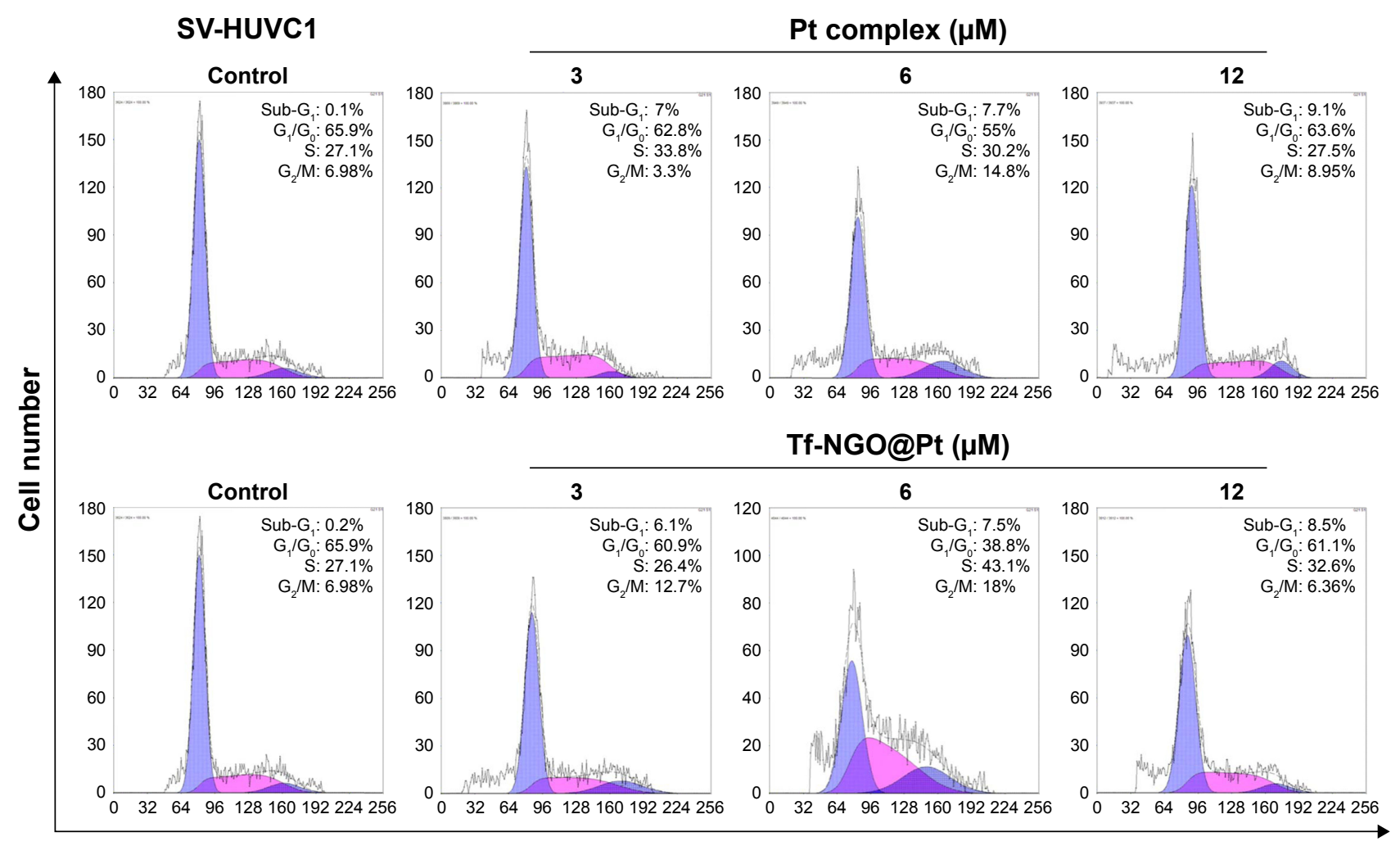

DNA content

Figure S4 Induction of cancer-cell apoptosis by Tf-NGO@Pt and flow-cytometry analysis of Pt complex and Tf-NGO@Pt-treated SV-HUVCI cells for 72 hours. Abbreviations: Tf, transferrin; NGO, nanographene oxide.

International Journal of Nanomedicine

\section{Publish your work in this journal}

The International Journal of Nanomedicine is an international, peerreviewed journal focusing on the application of nanotechnology in diagnostics, therapeutics, and drug delivery systems throughout the biomedical field. This journal is indexed on PubMed Central,

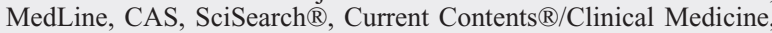

\section{Dovepress}

Journal Citation Reports/Science Edition, EMBase, Scopus and the Elsevier Bibliographic databases. The manuscript management system is completely online and includes a very quick and fair peer-review system, which is all easy to use. Visit http://www.dovepress.com/ testimonials.php to read real quotes from published authors. 